University of New Hampshire

University of New Hampshire Scholars' Repository

Space Science Center

Institute for the Study of Earth, Oceans, and

Space (EOS)

1997

\title{
5 years of Crab Pulsar observations with COMPTEL
}

\author{
R Much \\ ESTEC \\ K Bennett \\ ESTEC \\ C Winkler \\ ESTEC \\ R Diehl \\ Max-Planck-Institut für extraterrestriche \\ G G. Lichti \\ Max-Planck-Institut für extraterrestrische Physik
}

See next page for additional authors

Follow this and additional works at: https://scholars.unh.edu/ssc

Part of the Astrophysics and Astronomy Commons

\section{Recommended Citation \\ 5 years of Crab Pulsar observations with COMPTEL Much, R. and Bennett, K. and Winkler, C. and Diehl, R. and Lichti, G. and Schönfelder, V. and Steinle, H. and Strong, A. and Varendorff, M. and Hermsen, W. and Kuiper, L. and van der Meulen, R. and Connors, A. and McConnell, M. and Ryan, J. and Buccheri, R., AIP Conference Proceedings, 410, 542-546 (1997), DOI:http://dx.doi.org/10.1063/1.54127}

This Conference Proceeding is brought to you for free and open access by the Institute for the Study of Earth, Oceans, and Space (EOS) at University of New Hampshire Scholars' Repository. It has been accepted for inclusion in Space Science Center by an authorized administrator of University of New Hampshire Scholars' Repository. For more information, please contact Scholarly.Communication@unh.edu. 
Authors

R Much, K Bennett, C Winkler, R Diehl, G G. Lichti, V Schonfelder, H Steinle, A W. Strong, M Varendorff, W Hermsen, L Kuiper, R van der Meulen, A Connors, Mark L. McConnell, James M. Ryan, and R Buccheri 


\section{AIP $\mid$ proceedings}

\section{5 years of Crab Pulsar observations with COMPTEL}

R. Much, K. Bennett, C. Winkler, R. Diehl, G. Lichti, V. Schönfelder, H. Steinle, A.

Strong, M. Varendorff, W. Hermsen, L. Kuiper, R. van der Meulen, A. Connors, M.

McConnell, J. Ryan, and R. Buccheri

Citation: AIP Conference Proceedings 410, 542 (1997); doi: 10.1063/1.54127

View online: http://dx.doi.org/10.1063/1.54127

View Table of Contents:

http://scitation.aip.org/content/aip/proceeding/aipcp/410?ver=pdfcov

Published by the AIP Publishing

Articles you may be interested in

HESS Observations of Pulsar Wind Nebulae

AIP Conf. Proc. 801, 298 (2005); 10.1063/1.2141885

Gamma-rays from the Crab Nebula and other plerions-testing MHD models AIP Conf. Proc. 558, 154 (2001); 10.1063/1.1370788

Observation of the Crab Pulsar with BeppoSAX: study of the pulse profile and phase resolved spectroscopy

AIP Conf. Proc. 410, 553 (1997); 10.1063/1.54128

The infrared to gamma-ray pulse shape of the Crab Nebula pulsar

AIP Conf. Proc. 410, 547 (1997); 10.1063/1.54014

COMPTEL gamma-ray study of the Crab nebula

AIP Conf. Proc. 410, 537 (1997); 10.1063/1.54013 


\title{
5 years of Crab Pulsar observations with COMPTEL
}

\author{
R.Much $^{4}$, K. Bennett ${ }^{4}$, C. Winkler ${ }^{4}$, R. Diehl ${ }^{1}$, G. Lichti ${ }^{1}$, V. \\ Schönfelder ${ }^{1}$, H. Steinle ${ }^{1}$, A. Strong ${ }^{1}$, M. Varendorff ${ }^{1}$, W. \\ Hermsen $^{2}$, L. Kuiper ${ }^{2}$, R. van der Meulen ${ }^{2}$, A. Connors ${ }^{3}$, M. \\ McConnell ${ }^{3}$, J. Ryan ${ }^{3}$, R. Buccheri ${ }^{5}$
}

\footnotetext{
${ }^{1}$ Max-Planck Institut für Extraterrestrische Physik, P.O. Box 1603, 85740 Garching, F.R.G.

${ }^{2}$ SRON-Utrecht, Sorbonnelaan 2, NL-3584 CA Utrecht, the Netherlands

${ }^{3}$ Space Science Center, Univ. of New Hampshire, Durham NH 038\%4, U.S.A.

${ }^{4}$ Astrophysics Division, ESTEC, P.O. Box 299, NL-2200 AG Noordwijk, the Netherlands

${ }^{5}$ IFCAI/CNR, Piazza G. Verdi 6, 90139 Palermo, Italy
}

\begin{abstract}
Using the COMPTEL data of the first 5 years of the CGRO mission we have derived the average pulsed spectrum of the Crab Pulsar, as well as phase-resolved spectra of the pulsed emission of the Crab Pulsar. The spectra in the COMPTEL energy range (0.75 to $30 \mathrm{MeV})$ are compared to those in the neighbouring energy bands. The pulsed flux has been examined for its stability. Pulsed lightcurves have been derived for different energy intervals. Preliminary results of this analysis are presented.
\end{abstract}

\section{INTRODUCTION}

For our analysis of COMPTEL data we have selected all Crab viewing periods (VPs) out of the first 5 years of the CGRO operation, where the Crab Nebula was within $30^{\circ}$ of the pointing direction. In total there are 19 viewing periods, namely: $0,1,31,36,36.5,39,213,221,310,321.1321 .5,337,412$, $413,419.1,419.5,420,426,502$. This adds up to a total exposure of the Crab Nebula of $1.09 \cdot 10^{9} \mathrm{~s} \mathrm{~cm}^{2}$ in the $1-3 \mathrm{MeV}$ energy band.

\section{ANALYSIS}

The timing analysis was performed with the pulsar analysis subsystem of the COMPTEL Processing and Analysis Software System (COMPASS) using contemporary Crab radio observations (Arzoumanian et al. 1992). Lightcurves 

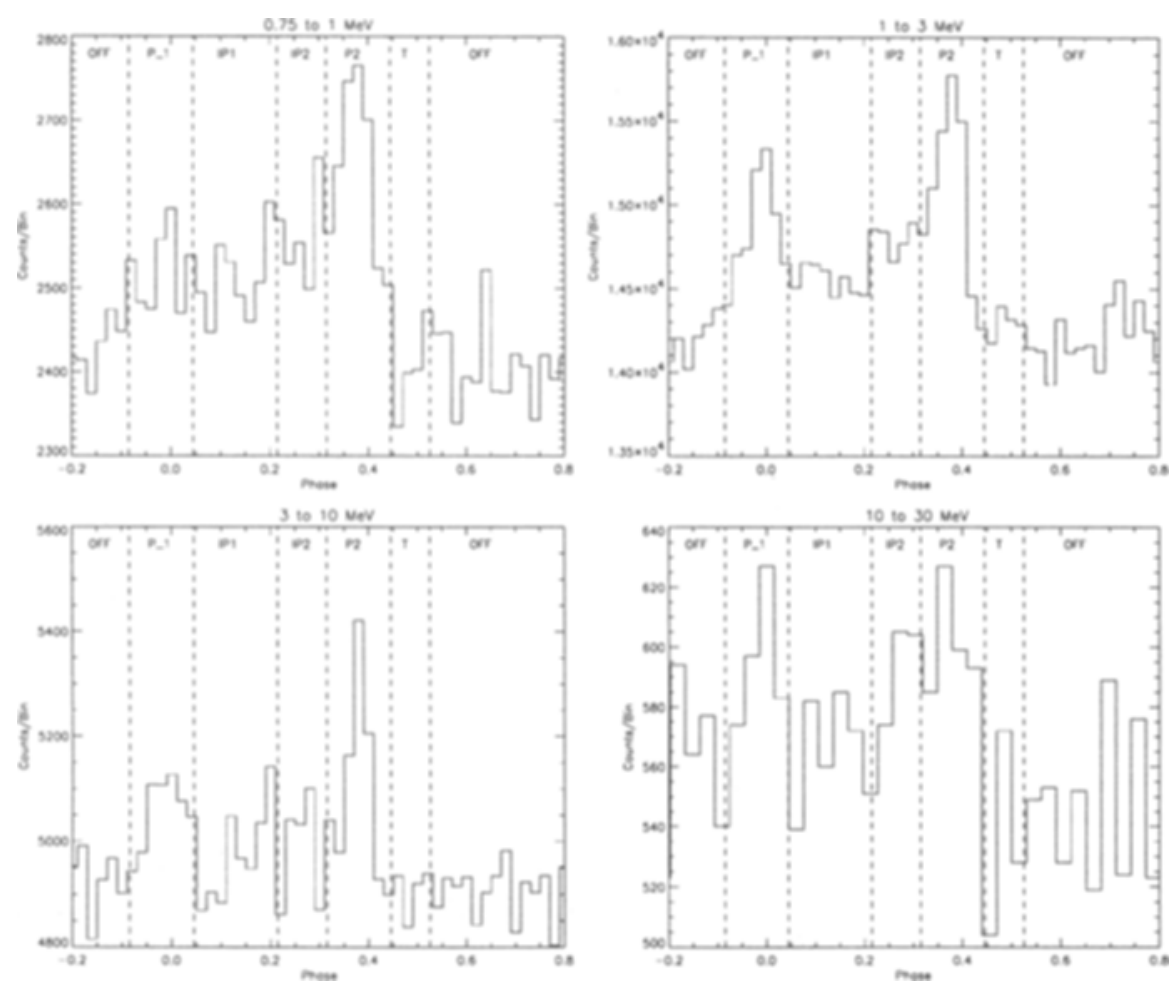

FIGURE 1. The Crab lightcurves of the combined observations for the four standard energy intervals.

were created for the combined observations by folding the photon arrival times with the pulsar period once they were transformed to the Solar System Barycentre.

We used a maximum likelihood ratio method to calculate the source flux with its associated statistical uncertainty (Bloemen et al. 1994). COMPTEL cannot resolve the Crab pulsar from the surrounding nebula. Therefore we used phase selection to disentangle the unpulsed and pulsed emission assuming that the unpulsed emission remains constant over the full pulsar period.

The pulsed emission $\Phi_{\Delta_{p}}$ was determined by calculating the total (i.e. pulsed plus unpulsed) flux $\left(\Phi_{\text {total }}\right)_{\Delta_{p}}$ in a pulsed phase interval $\Delta_{p}$ with subsequent subtraction of the unpulsed component $\Phi_{\Delta_{u p}}$ :

$$
\Phi_{\Delta_{p}}=\left(\Phi_{\text {total }}\right)_{\Delta_{p}}-\Phi_{\Delta_{u p}} \cdot \frac{\Delta_{p}}{\Delta_{u p}}
$$

where $\Delta_{p} / \Delta_{u p}$ is the ratio of the width of the pulsed phase to the width of the unpulsed phase interval.

Phase-resolved photon spectra of the pulsed emission were calculated for the 


\begin{tabular}{l|c|c|c|c} 
Phase & $\begin{array}{c}\text { Phase } \\
\text { Interval }\end{array}$ & $\begin{array}{c}I_{0} \\
10^{-4} \mathrm{ph} / \mathrm{cm}^{2} / \mathrm{MeV}\end{array}$ & $\alpha$ & $\chi^{2}$ (5 dof $)$ \\
\hline P1 & $0.915-0.445$ & $2.59 \pm 0.41$ & $-2.22 \pm 0.18$ & 7.2 \\
IP1 & $0.045-0.215$ & $1.89 \pm 0.45$ & $-2.65 \pm 0.35$ & 1.7 \\
IP2 & $0.215-0.315$ & $2.73 \pm 0.50$ & $-2.63 \pm 0.35$ & 3.1 \\
P2 & $0.315-0.445$ & $4.57 \pm 0.43$ & $-2.31 \pm 0.13$ & 3.8 \\
tot. pulsed & $0.445-0.525$ & $2.52 \pm 0.28$ & $-2.37 \pm 0.15$ & 1.6
\end{tabular}

TABLE 1. Results of powerlaw fitting of the 5-year averaged spectra. All fluxes are instantaneous.

smaller phase intervals indicated in Fig. 1. The phase definitions used earlier by Nolan et al. (1993) and Much et al. (1995) were adopted.

\section{LIGHTCURVES}

Figure 1 shows the lightcurves we obtained for the combined observations in the four COMPTEL standard energy intervals $(0.75$ to $1 \mathrm{MeV}, 1$ to $3 \mathrm{MeV}$, 3 to $10 \mathrm{MeV}$ and $10 \mathrm{MeV}$ to $30 \mathrm{MeV}$ ). The lightcurves show two peaks with a phase separation of 0.4 . Compared to energies above $50 \mathrm{MeV}$ (Nolan et al., 1993), there is relatively significant interpulse emission. The peak intensities change within the COMPTEL energy band relative to each other. Towards higher energies the intensity of the first peak increases relative to the second peak. This is to be expected considering the shape of the lightcurve measured above $30 \mathrm{MeV}$ by EGRET, where the first peak dominates.

\section{SPECTRAL ANALYSIS}

All derived COMPTEL spectra (both total pulsed and phase-resolved), when fitted alone, can be described with a simple powerlaw, $I_{0} / 1.65 \mathrm{MeV} \cdot E^{\alpha}$. Our fit results are listed in Table 1.

\section{Total Pulsed Spectrum}

The total pulsed emission for the COMPTEL standard energy intervals was calculated for each VP. For the interval with the best statistics (1-3 MeV) the Crab pulsar's emission is shown as a funtion of time (TJD) in Fig. 2. The flux is compatible with a constant flux, except for the combination of VP $412+413$, where we only received an upper limit. Further analysis of these VPs are required.

The spectrum of the total pulsed emission is shown in Fig. 3 as measured by 


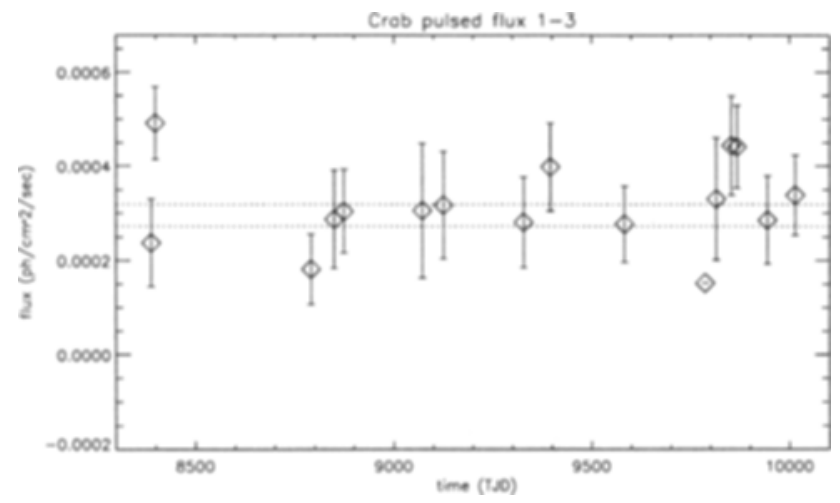

FIGURE 2. Instantaneous flux of the Crab pulsar in the 1-3 MeV energy band measured in the different VPs. The two horizontal lines indicate the $\pm 1 \sigma$ uncertainty of the average flux in the entire period. The data point without error-bar is an upper limit (sum of VPs $412+413)$.

OSSE, COMPTEL and EGRET. However the OSSE, EGRET and COMPTEL spectra are not contemporaneous. Nevertheless the total pulsed spectra as measured by OSSE, COMPTEL and EGRET connect smoothly.

\section{Phase-Resolved Spectra}

The phase-resolved spectra for the first peak (P1), first and second interpulse (IP1 and IP2) and for the second peak (P2) were calculated. We compared the COMPTEL spectrum with the EGRET spectra obtained by Nolan et al (1993). The data have to be interpreted carefully, as the EGRET data are not contemporaneous with the COMPTEL data. However the constancy of the COMPTEL total pulsed flux (Fig 2.) and the smooth connection of the total pulsed spectrum (Fig 3.) suggest that a comparison is valid.

Although our results are preliminary (e.g. the P1 spectrum requires further analysis), the following conclusions can already be drawn from the phaseresolved spectra.

- the COMPTEL P2 spectrum smoothly connects to EGRET

- the IP1 and IP2 spectra clearly require a change in slope between the COMPTEL and the EGRET data

\section{SUMMARY}

- In the COMPTEL energy range the ratio of the intensities of the two main peaks of the lightcurve changes, namely, with increasing energy the first peak becomes more dominant relative to the second peak. 
- The total pulsed emission can be described by a single powerlaw

- Combining the COMPTEL with the EGRET phase-resolved spectrum shows that

- the phase-resolved spectrum of the second pulse connects smoothly to EGRET

- the IP1 and IP2 spectra require a change in slope between the COMPTEL and the EGRET data

\section{ACKNOWLEDGMENTS}

The COMPTEL project is supported by the German government through DARA grant 50 QV 90968 , by NASA under contract NASS-26645 and by the Netherlands Organisation for Scientific Research (NWO).

\section{REFERENCES}

1. Arzoumanian Z., et al., 1992, GRO/radio timing data base, Princeton University

2. Bloemen, H., et al., 1994, A\&AS 92, 419

3. Much, R., et al., 1995, A\&A, 299, 435

4. Nolan, P., et al., 1993, ApJ, 409, 697

5. Ulmer, M., et al., 1994, ApJ, 432, 228

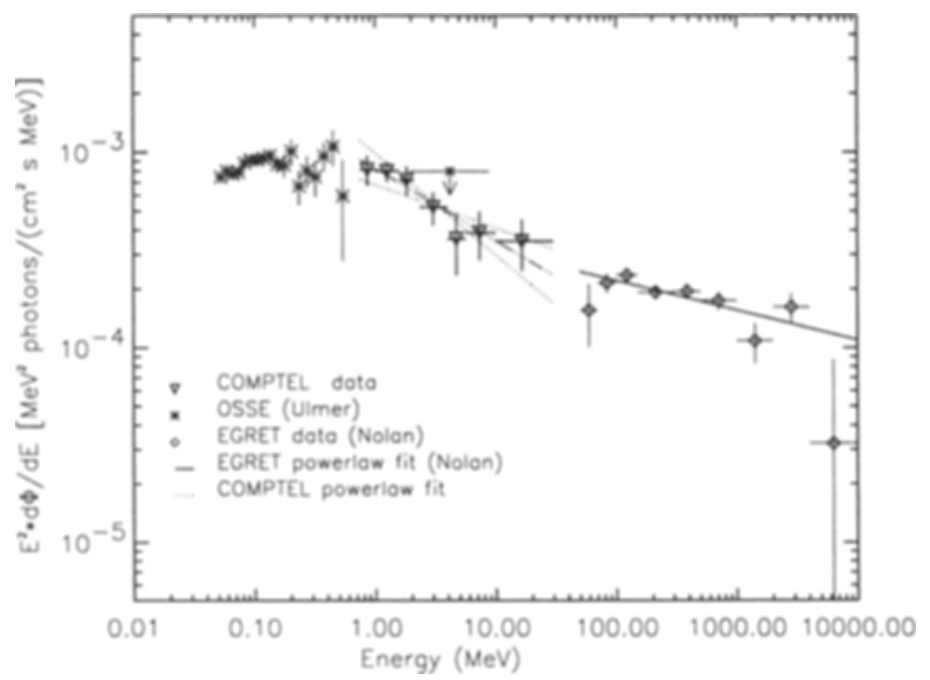

FIGURE 3. Instantaneous spectrum of the Crab total pulsed emission of the combined observations. The OSSE and EGRET spectra shown are not contemporaneous. Nevertheless the spectra of the three instruments connect smoothly. 